\title{
The Myths and Truths About Comparing Syndromic Data Across Sites
}

\section{Michael Coletta* and Aaron Kite-Powell}

CSELS/DHIS, Centers for Disease Control and Prevention, Atlanta, GA, USA

\section{Objective}

As the BioSense Platform matures and more sites submit surveillance data, many in the community have voiced concerns about comparing data across sites. Recently, a number of jurisdictions from across the country were asked to provide opioid overdose data to a news agency highlighting the epidemic. Many jurisdictions requested information on how to present syndromic surveillance data from across sites and shared concern about how the data would be interpreted. This round table will address those concerns and explore options for comparing data across sites.

\section{Introduction}

One of the more recent successes of NSSP has been the introduction of more robust data quality monitoring and reporting. However, despite the increased insight into data quality, there are still concerns about data sharing and comparisons across sites. For NSSP to be most effective, users need to feel confident in sharing data and making comparisons across sites.

\section{Keywords}

NSSP; Sharing Data; Comparing Data Across sites; National Surveillance; Regional Surveillance

\section{*Michael Coletta}

E-mail: mac0@cdc.gov 